\title{
Thrombolysis in Myocardial Infarction Flow
}

National Cancer Institute

\section{Source}

National Cancer Institute. Thrombolysis in Myocardial Infarction Flow. NCI Thesaurus.

Code C100021.

A grading system for coronary blood flow based on the classification developed by the Thrombolysis in Myocardial Infarction Group. It classifies coronary blood flow into four classes based upon the angiographic appearance of the blood vessels. (ACC) 